\title{
The Effects of Egg Windowing on the Viability and Longevity of Gallus gallus Embryos in an Undergraduate Teaching Lab
}

\author{
Rebekah A. Hobbs ${ }^{1,2}$ and Jeffrey O. Henderson ${ }^{1,2,3}$ \\ ${ }^{1}$ Department of Science and Mathematics, Judson University, Elgin, IL, USA \\ ${ }^{2}$ Equal contribution \\ ${ }^{3}$ Corresponding author: jeffrey.henderson@judsonu.edu
}

\section{$\underline{\text { ABSTRACT }}$}

The observation and manipulation of chicken embryos in ovo has been useful for understanding the development of vertebrates. However, the viability and longevity of the embryos are severely compromised even by simple manipulations to the egg shell. We have explored experimental protocols that promote the viability of embryos in ovo and ex ovo for use in an undergraduate teaching laboratory setting. Here, we demonstrate that a modified in ovo windowing technique increases survival time over an ex ovo method but with concomitant loss of spatial and temporal examination of chick embryo development.

\section{Introduction}

Developmental studies involving the windowing of fertilized chicken (Gallus gallus) eggs have been used since the middle of the twentieth century (Auerbach et al., 1974). However, this method of observation drastically decreases the temporal viability of the embryo in gestation and limits research time for pedagogical purposes. Many modifications have been done to the windowing technique with the objective to increase the number of days that the embryo is alive once the shell has been compromised (Andacht et al., 2004). Increased viability leads to more time for manipulation of embryos to gain a better understanding of development.

Keller and coworkers (1999) describe a methodology where embryos are windowed two days post-incubation and observed for two weeks. Preliminary data generated in our laboratory using the method outlined by Keller indicates that embryos survived for a maximum of five days post-windowing (unpublished data). This loss of embryos at a consistent period of gestation instigated further research for an experimental procedure to increase the viability of chicken embryos post-windowing. Several alternative procedures for windowing chicken eggs were found reporting similar high mortality rates (reviewed in Andacht et al., 2004). Numerous explanations for the high death rate were described, including the observation that exposure of the embryo to the exterior environment allows for the introduction of air bubbles into the albumen. The presence of air bubbles (specifically oxygen) is teratogenic and increases lethality resulting in low hatch rates. This observation suggests that an airtight seal is needed to increase viability (Andacht et al., 2004). Furthermore, Zareen and Shahid (2011) have reported that an incubation environment with 60$70 \%$ humidity is essential. This is contrary to our past protocol where humidity is not so finely controlled with levels between $45-65 \%$ (Henderson, unpublished data). The viability of embryos can be determined by the presence of engorged vasculature surrounding the embryo and embryonic movement in later stages of development. In this study several experimental protocols, two in ovo and one ex ovo, were used to determine the methodology that promotes longevity of chicken embryos during undergraduate lab observations. 


\section{Experimental Procedures}

\section{Chicken Egg Acquisition and Maintenance}

Gallus gallus eggs were ordered from Carolina Biological Supply Company (Burlington, NC). Upon arrival, eggs were stored at $15-20^{\circ} \mathrm{C}$ and turned twice daily until ready for windowing or incubation. This time period was never more than one week. The eggs were incubated in a Hova-Bator model 1583, having an automatic egg turner, at 98$100^{\circ} \mathrm{F}\left(36-38^{\circ} \mathrm{C}\right)$ and $45-65 \%$ humidity. After the eggs were windowed or cultured the egg turner was removed from the Hova-Bator before continuing incubation. Prior to manipulation eggs were cleaned with $70 \%$ ethanol and placed in a horizontal position for approximately ten minutes to allow the embryo to migrate to the top providing maximal viewing of embryogenesis. The USDA Animal Welfare Act excludes birds from regulatory oversight; therefore, our research did not require IACUC review/approval (https://www.aphis.usda.gov/animal_welfare/downloads/AC_BlueBook_AWA_508_comp_version.pdf). However, we consistently treated all chick embryos in this study humanely as described by the American Veterinary Medical Association. Thus, living embryos were euthanized by hypothermia at $-20 \mathrm{C}$ as prescribed by the American Veterinary Medical Association Panel on Euthanasia Section S3.4.4 on avian embryos and neonates (https:/www.avma.org/sites/default/files/2020-01/2020-Euthanasia-Final-117-20.pdf). To illustrate the numbers of eggs needed to perform experiments, we have included the numbers of eggs unfertilized ( $\sim 50 \%$; Tables 1 and 2$)$ and those lost to mechanical errors to give experimenters an ideas of how to plan the number of eggs to use in their studies. These rates had no effect on the differing windowing techniques except as a guide to how many eggs were needed to observe embryogenesis.

\section{Chicken Egg windowing I: "Korn Method"}

Eggs were windowed as described in Korn \& Kramer (2007). Specifically, after placing the egg in a horizontal position, an " $X$ " was marked on the top with a pencil (ink will kill the embryo) to denote where the relative position of the embryo would be within the egg. A razor blade and dissection scissors were disinfected with $70 \%$ ethanol and a $1 \times 1 \mathrm{~cm}$ piece of tape was cut from a roll of Scotch 3M191CL 1.5"x 125" plastic tape (ULine, Waukegan, IL). This piece of tape was placed slightly above the base of the egg (the wider end). The dissection scissors were then used to drill a small hole through this piece of tape until it made a needle sized hole in the base of the shell. A sterile $10 \mathrm{~mL}$ syringe with a 20 gauge needle was inserted vertically into the hole until it touched the bottom of the shell and then angled $45^{\circ}$. Approximately 3-4 $\mathrm{mL}$ of albumin was removed from within the shell to prevent the embryo on top from making contact with the membrane. The syringe was then removed and the hole was covered with a $1 \mathrm{x} 1 \mathrm{~cm}$ piece of tape. Next, a $10 \times 15 \mathrm{~cm}$ piece of tape was cut and stretched over the top of the egg where the " $\mathrm{X}$ " was placed. Care was taken to reduce the amount of wrinkles in the tape when stretched across the surface. Sterilized dissection scissors were used to drill a hole into the top of the shell. Once the shell had been pierced, the entire blade was inserted into the shell while angling the point of the blade upwards toward the top of the shell. A circular hole was cut into the top of the shell leaving the hole partially uncut to act as a hinge. After documentation of the embryo, the shell flap was secured back into place with a small piece of tape and placed into the Hova-Bator without the automatic turner. Eggs were viewed and recorded daily until disintegration of the blood vessels were observed indicating embryonic death.

\section{Chicken Egg Windowing II: "Andacht Method"}

Eggs were windowed according to Keller and colleagues (1999) with modifications (Andacht et al., 2008). After denotation of the top of the egg with an " $X$ " using a pencil, a $10.16 \mathrm{~cm}$ metal file and forceps were disinfected with $70 \%$ ethanol. The metal file was used to file a $2 \times 2 \mathrm{~cm}$ window on top of an egg that had been pre-incubated as indicated in Results and Discussion. Care was taken to only file through the shell leaving the membrane underneath intact. The top of the shell was periodically wiped with a Kimwipe ${ }^{\mathrm{TM}}$ to remove any debris that could possibly fall on top of the embryo. Forceps were used to remove the remaining shell and then used to detach the shell membrane starting from a corner of the window and peeling the membrane backwards. An 18x18 mm glass cover slip (Fisher Gold, Fisher Scientific) was swiftly placed on top of the window to reduce the amount of time the embryo is exposed to the 
atmosphere and hot glue from a 120V-40W low temperature glue gun was inserted into the space where the cover slip and the shell meet. After letting the glue harden for about one minute, the space between the shell and the cover slip was reinforced in areas where the glue was thin or there were gaps. Keller and coworkers (1999) suggest sealing the window by one of three methods: using a coverslip sealed with melted wax ("a permanent closure; may fog"), sealing the hole with parafilm ("a temporary closure; potential contamination"), or covering the hole with sticky tape ("a permanent closure; may fog"). We chose the method by Andacht et al. (2008) where a coverslip is secured using hot glue. We found that using a glue gun gave more precise handling than melted wax. Furthermore, fogging and contamination were both reduced by this modification (data not shown).

\section{Ex Ovo Embryo Culturing}

Eggs were pre-incubated and turned in the Hova-Bator for the indicated time in the Results and Discussion. Eggs were disinfected and oriented as described above under Chicken Egg Windowing I: Korn Method. Next, the shell was cracked and the embryo, albumin, and extraembryonic membranes carefully emptied into a $100 \mathrm{x} 15 \mathrm{~mm}$ sterile plastic petri dish or a $100 \times 20 \mathrm{~mm}$ sterile glass $P y r e{ }^{\circledR}$ petri dish. If the embryo made it into the petri dish without rupturing the yolk sac, the petri dish was placed inside a Nunc ${ }^{\circledR} 150$ x $25 \mathrm{~mm}$ sterile plastic petri dish. The larger petri dish was then filled with sterile water until the bottom was completely covered in water $(\sim 30 \mathrm{~mL})$. The embryo was then placed into the Hova-Bator or a $38^{\circ}$ bacteriological incubator (ambient humidity) and monitored daily.

\section{Results and Discussion}

\section{Chicken Egg Windowing I: "Korn Method"}

At the beginning of this experiment, we divided 30 eggs into three groups. The non-incubation group consisted of 9 eggs and were windowed prior to any time spent at $36-38^{\circ} \mathrm{C}$; the pre-incubation groups were windowed after two (12 eggs) or five days ( 9 eggs) of incubation at $36-38^{\circ} \mathrm{C}$ to determine if there is a difference in viability.

After 24 hours post-windowing, the pre-incubation group showed large blastodiscs, indicating cell division (Figure 1A). After 79 hours, all embryos (pre- and non-incubated) were showing signs of angiogenesis (data not shown). By the seventh day of gestation, all embryos were dead (Table 1), with the yolk exhibiting a dark yellow color and a rubbery texture as seen in Figure 1B.
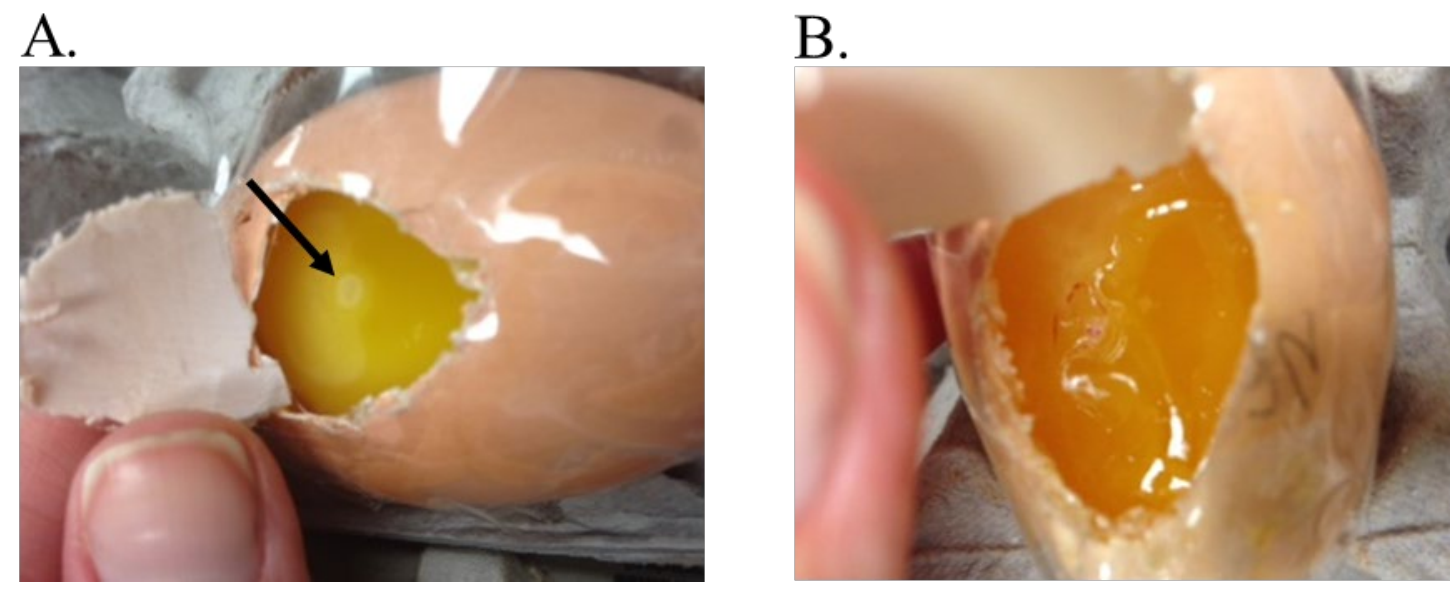

Figure 1. Effects of the Korn method on egg contents (A) Pre-incubated eggs show a larger blastodisc than those not pre-incubated. The enlarged blastodisc is indicated by the black arrow. (B) Insufficient humidity leads to desiccation of the albumin and yolk as indicated by the rubbery appearance after 48 hrs. of incubation. 
Table 1

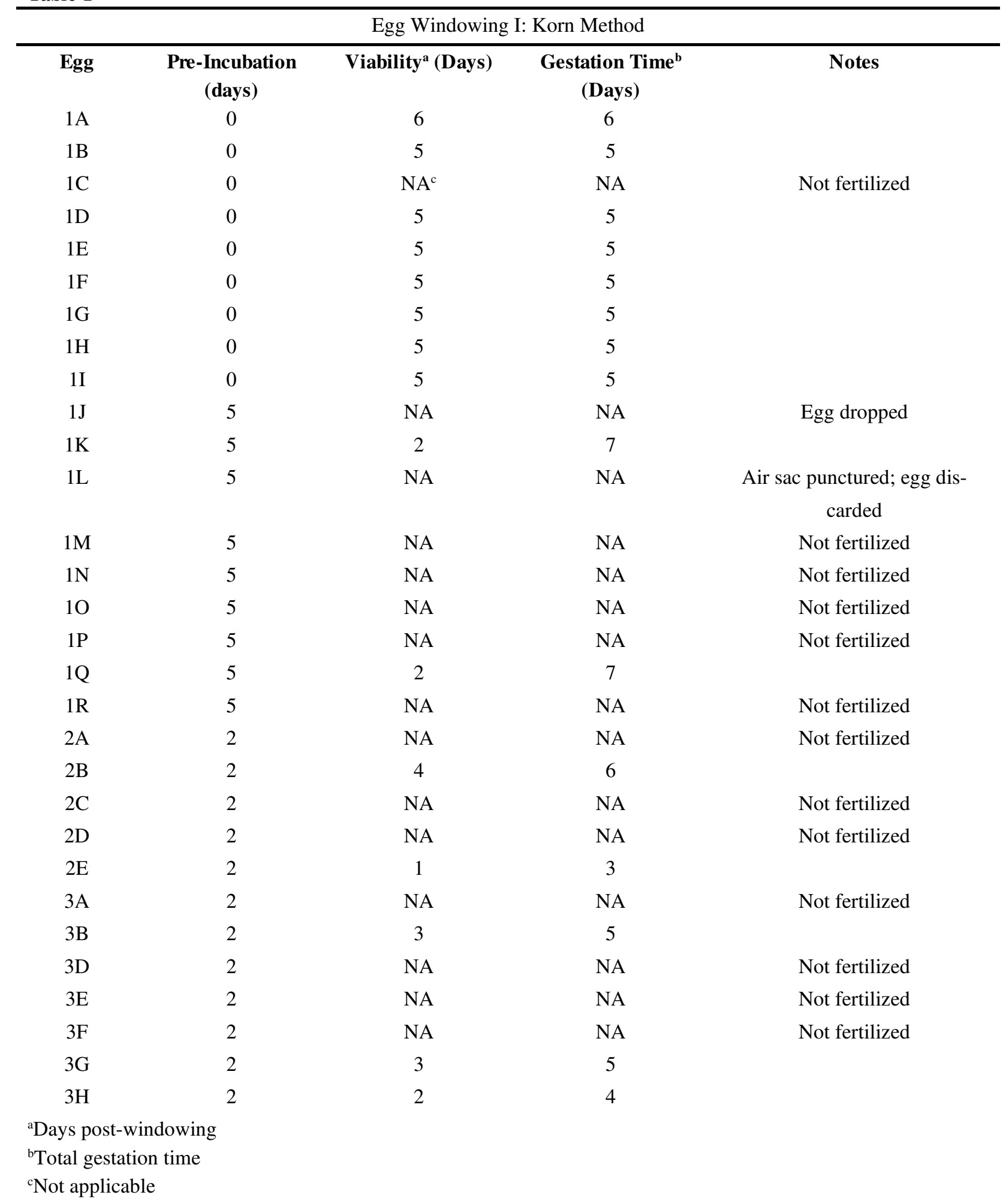

Only seven of the twenty-one pre-incubation eggs made it through the windowing process. Twelve were not fertilized; two were lost to mechanical errors such as puncturing the air sac with the syringe or being dropped. The seven preincubation eggs that survived windowing died after 48-96 hours post-windowing (Table 1). These results indicate that 
the embryos from eggs windowed prior to incubation lived longer than the eggs incubated prior to manipulation (Table 1, Viability). However, when calculating the full gestation time from the beginning of incubation to death, the preincubation group lived up to one more day than the non-incubation group.

In eggs windowed using the Korn method, the embryos, and surrounding membranes (e.g. yolk sac), often looked dry and rubbery upon embryonic death (Figure 1B). We attributed this to dehydration from the fluid portion of the albumin through the open and unsealed window. Additionally, during the windowing process, many eggs developed cracks in the shell that extended beyond the plane of the window. This makes the embryo unable to continue proper development and they were discarded (Korn \& Cramer, 2007). When an embryo is exposed to the outer environment air bubbles form in the albumen. These air bubbles (specifically oxygen) are teratogenic and lethal resulting in low hatch rates (Andacht et al., 2004). This windowing technique is reported to produce embryos with long survival times that extend through advanced embryonic stages (Zareen and Shahid, 2011). In contrast, we observed that most of the embryos would develop a heartbeat, a defined A-P axis, and a brain, yet would not develop past the fifth day of gestation (Figure 2 and Table 1).

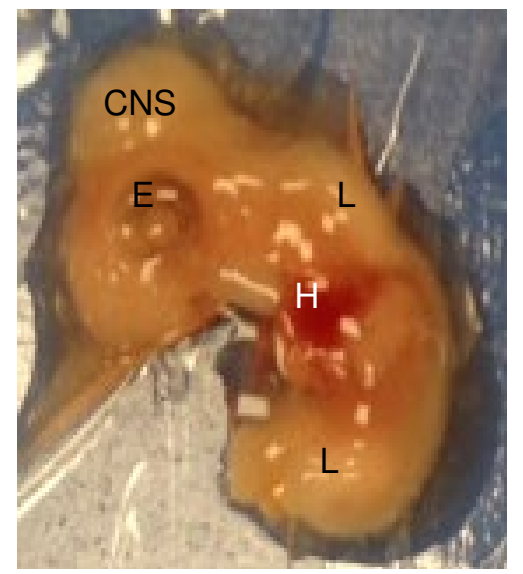

Figure 2. Embryonic development using the Korn method. Gestational stage (day 5) that majority (8/15) of embryos would develop to and then die as observed after removal from shell. Note the prominent eye (E), heart $(\mathrm{H})$, limb buds (L), and central nervous system (CNS).

\section{Chicken Egg Windowing II: "Andacht Method"}

The methodology outlined by Andacht, Hu and Ivarie (2008) reported an increase in hatchability from 32\% to $63 \%$ because sealing the window prevents the entrance of air bubbles toxic to the embryo. The expectation was that with careful manipulation, the total gestation time before death of our embryos would increase as well. Our data show that the longevity of our embryos had a statistically significant increase (Figure 3) from an average of 5.2 days using the Korn method to 10.4 days with the Andacht method $(p=0.0001)$. However, this increase is in combination with longer pre-incubation times. The range of days that the embryo survived after windowing, even with a sealed window, was no different than the method given by Korn and Cramer (2007; compare Viability in Table 1 vs. Table 2). Embryos were counted if they successfully made into the incubator without any mechanical errors. Some of the eggs that were windowed after day seven had blood vessels sticking to the inside of the membrane (not shown). When the membrane was peeled back or the shell was windowed some of these blood vessels would burst causing a few of the embryos to die by exsanguination. We discovered that this could be prevented if the embryo was candled for the presence of vasculature and then windowed on the opposite side of the blood vessels. The viability of the embryo was based on the presence of vasculature and the recorded days of viability were counted after windowing. This methodology allowed for longer gestation when compared to the Korn method (2007). There was a positive relationship between the number of pre-incubation days and the days that the embryo was viable. The embryos lived significantly longer if they were windowed around day nine of gestation as opposed to day seven: $3 \mathrm{~N}, 3 \mathrm{Q}$, and $3 \mathrm{R}$ were pre-incubated for 9 days 
and lived 16 gestational days. That is, these embryos survived seven days post-windowing. In contrast, the embryos incubated for seven days only lived a gestation of nine days (Table 2). One embryo, 3U, survived past sixteen days of gestation to 19 days gestational age. This embryo was pre-incubated 12 days and lived 7 days post-windowing a similar length to those embryos pre-incubated 9 days. Because of the small window that embryo $3 \mathrm{U}$ was observable through (not shown), pictures could not be taken until after euthanizing the embryo and removing it from the shell. However, as it developed in ovo and would move we were able to see small glimpses of feathers, scales, and toes. After being euthanized and removed from the shell we observed that the embryos gastrointestinal system was outside of its body, but it had developed all major organ systems (Figure 4). In chick development, the gut recedes from the yolk sack and into the body after day nineteen of gestation (Schoenwolf, 2009) confirming that this embryo was indeed at or around day 19 of gestation.

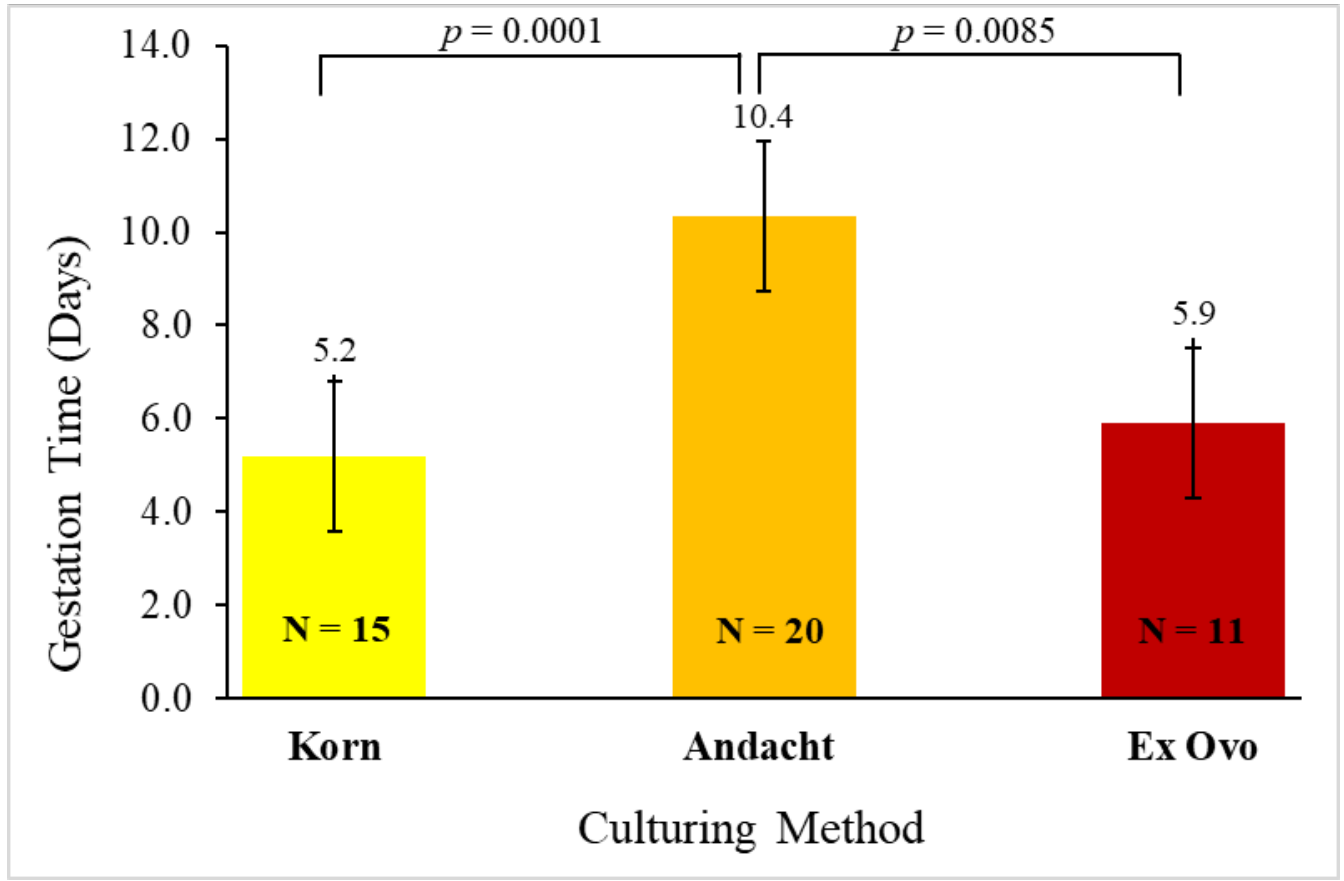

Figure 3. Comparison of gestation times by methodology. Mean days of gestation are listed above each bar. Standard deviation around the mean is indicated by the error bar; number of embryos $(\mathrm{N})$ observed is indicated.

Table 2

\begin{tabular}{cccc}
\hline Egg & $\begin{array}{c}\text { Egg Windowing II: Andacht Method } \\
\text { Pre-Incubation } \\
\text { (days) }\end{array}$ & Viability $^{\mathbf{a}}$ (Days) & $\begin{array}{c}\text { Gestation Time }^{\mathbf{b}} \\
\text { (Days) }\end{array}$ \\
3C & 2 & 3 & 5 \\
3I & 2 & 2 & 4 \\
$3 \mathrm{~J}$ & 2 & 2 & 4 \\
$3 \mathrm{~K}$ & 7 & 2 & 9 \\
$3 \mathrm{~L}$ & 7 & 2 & 9 \\
$3 \mathrm{M}$ & 7 & 2 & 9 \\
$3 \mathrm{~N}$ & 9 & 7 & 16 \\
$3 \mathrm{O}$ & 9 & 2 & 11
\end{tabular}




\begin{tabular}{|c|c|c|c|c|}
\hline $3 \mathrm{P}$ & 9 & $\mathrm{NA}^{\mathrm{c}}$ & NA & $\begin{array}{l}\text { Mechanical error; egg dis- } \\
\text { carded }\end{array}$ \\
\hline $3 \mathrm{Q}$ & 9 & 7 & 16 & \\
\hline $3 R$ & 9 & 7 & 16 & \\
\hline $3 \mathrm{~S}$ & 9 & NA & NA & $\begin{array}{c}\text { Mechanical error; egg dis- } \\
\text { carded }\end{array}$ \\
\hline $3 \mathrm{~T}$ & 12 & 4 & 16 & \\
\hline $3 \mathrm{U}$ & 12 & 7 & 19 & \\
\hline $3 \mathrm{~V}$ & 12 & 4 & 16 & \\
\hline $4 A-4 E$ & 5 & NA & NA & Not fertilized \\
\hline $4 \mathrm{~F}$ & 5 & 2 & 7 & \\
\hline $4 \mathrm{G}$ & 5 & NA & NA & Not fertilized \\
\hline $4 \mathrm{H}$ & 5 & 2 & 7 & \\
\hline $4 \mathrm{I}$ & 5 & NA & NA & Not fertilized \\
\hline $4 \mathrm{~J}$ & 5 & 4 & 9 & \\
\hline $4 \mathrm{~K}-4 \mathrm{~L}$ & 5 & NA & NA & Not fertilized \\
\hline $5 \mathrm{~A}-\mathrm{L}$ & 5 & NA & NA & Not fertilized \\
\hline $6 \mathrm{~A}$ & 5 & 3 & 8 & \\
\hline $6 \mathrm{~B}$ & 5 & 5 & 10 & \\
\hline $6 \mathrm{C}$ & 5 & 3 & 8 & \\
\hline $6 D$ & 19 & $N A$ & 19 & Control for 19 day gestation \\
\hline $6 \mathrm{E}$ & 5 & NA & NA & Embryo Dead upon windowing \\
\hline $6 \mathrm{~F}$ & 5 & NA & NA & Not fertilized \\
\hline $6 \mathrm{G}$ & 2 & NA & NA & Not fertilized \\
\hline $6 \mathrm{H}$ & 2 & NA & NA & Not fertilized \\
\hline $6 \mathrm{I}$ & 2 & 6 & 8 & \\
\hline $6 \mathrm{~J}$ & 2 & NA & NA & Not fertilized \\
\hline $6 \mathrm{~K}$ & 2 & NA & NA & Not fertilized \\
\hline $6 \mathrm{~L}$ & 2 & NA & NA & $\begin{array}{l}\text { Mechanical error; egg dis- } \\
\text { carded }\end{array}$ \\
\hline \multicolumn{5}{|c|}{$\begin{array}{l}\text { aDays post-windowing } \\
{ }^{\mathrm{b}} \text { Total gestation time } \\
{ }^{\mathrm{c}} \text { Not applicable }\end{array}$} \\
\hline
\end{tabular}




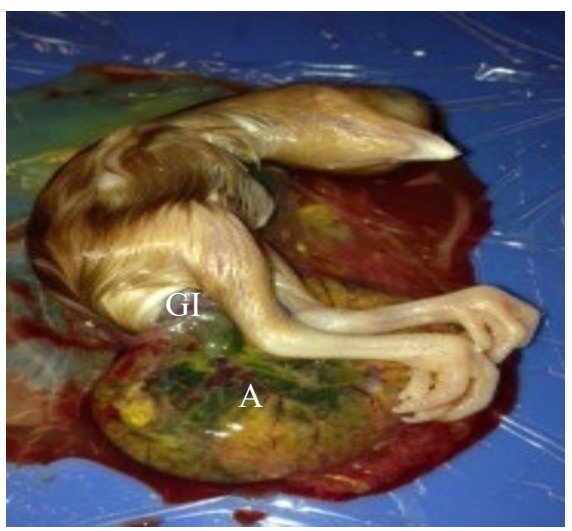

Figure 4. Antepenultimate gestation of embryo windowed by the Andacht method. Embryo $3 \mathrm{U}$ at approximately 19 days of gestation; note the gastrointestinal (GI) system is located outside of the body adjacent to the allantois (A).

The fifth group of eggs were windowed with the same methodology after a pre-incubation period of two days. None of the eggs showed signs of viability, let alone, any indication that they were fertilized. In group 6, 6A-F were preincubated for five days and 6G-K were pre-incubated for two days (Table 2). Except for egg 6I, eggs 6G-K showed no signs of growth or fertilization. Embryo 6I lived for a total of eight days. After two days of pre-incubation it remained viable for six days; this was longer than any other embryo that had been pre-incubated for the same amount of time. Egg 6E had been fertilized, but the embryo was found to be dead upon windowing. Egg 6F had not been fertilized and was discarded. These data indicate that longer pre-incubation times lead to increased viability postwindowing and longer gestation times as exemplified by the following embryos: 3N, 3Q, 3R, 3T, 3U, and 3V (Table 2). This phenomenon is most likely due to protection of the embryo by the egg shell during the most sensitive times of early development in which organogenesis occurs (Gilbert, 2010; Schoenwolf, 2009).

\section{Ex Ovo Embryo Culturing: "Auerbach Method"}

Three dozen eggs were used by us to perform ex ovo manipulations. We began with candling the eggs to look for vasculature as signs of fertilization after 72 hours of pre-incubation as outlined in Schoenwolf (2009). This candling technique resulted in eggs being mistaken for unfertilized ones due to our inexperience in recognizing small embryos and scant vasculature through the shell. In an undergraduate teaching lab where students are most likely handling fertilized eggs for the first time we recommend all eggs be handled as if they are inseminated to reduce unnecessary loss and optimize yield. Only those embryos that made it into an incubator having intact extraembryonic membranes (e.g. yolk sac) and a beating heart were recorded (Table 3). All losses reported are primarily due to the breaking of the yolk sac upon cracking of the shell making the embryo nonviable (Auerbach et al., 1974); as such, those embryos were discarded. Furthermore, any egg that was not in the optimal orientation would die faster. The optimal orientation for the embryo would be the yolk sac near the center of the petri dish with the embryo lying directly on top and centered (Figure 5). For example, embryo 7B was on the edge of the yolk sac with part of the vasculature under the yolk sac and was found dead within 48 hours. As opposed to embryo 7A that lived 72 hours and was situated closer to the center of the yolk (data not shown but see Figure 5).

Due to the embryo being outside of the shell, the ex ovo methodology outlined by Auerbach and coworkers (1974) provided clearer temporal and spatial observations of the embryos (compare Figures 5 and 1) but with the same average gestation time as the Korn method and a statistically significant lower gestation time than the Andacht method $(p=0.0085$; Figure 3).

The incubator that was used for embryos 8D and 9A was not humidity controlled. Sterile water was added daily; however, the embryos were both found dead within 24 hours. These data amplify the need for strictly controlled humidity (See Experimental Procedures). Embryo 7C lived thirteen days post-culturing and sixteen gestational days including the three days of pre-incubation. This was the longest any embryo had lived with that short of a pre- 
incubation time (Compare Table 3 to Tables $1 \& 2$ ). The animal developed a beak, feather, and toes (i.e. complete organogenesis), yet it was "flimsy" (Figure 5). This "flimsiness" was caused by a reduction in skeletal mineralization due to absence of large quantities of $\mathrm{Ca} 2+$ from the shell for bone growth (Gilbert, 2010). As embryo 7C grew its head began to adhere to the inside of the petri dish lid (not shown) suggesting that there was limited space for its development. Unfortunately, the petri dishes being used were $5 \mathrm{~mm}$ shorter than recommended (Schoenwolf, 2009).

Based on the protocol from Schoenwolf (2009), $80 \%$ of the embryos used for culturing were expected to make it into the incubator. However, in our hands, upon cracking the shell, the yolk sac broke in $66 \%$ of the eggs used (24/36) with only 12 of 36 embryos (33\%) making it into the incubator (Table 3). Breaking of the yolk sac upon removal from the egg results in premature embryonic death. In the case of embryo 7D, the yolk sac seemed intact upon initial placement into the culture dish, but was later found to be ruptured. This was an expected phenomenon (Auerbach, 1974). A 50\% loss of embryos was anticipated within the first three days. We observed a loss of $91 \%$ (i.e. only one embryo, 7C, survived past 3 days; Table 3) due to our inexperience in performing these manipulations (Table 3). This was the case even though we exceeded the $80 \%$ threshold of success when practicing on unfertilized eggs purchased from a local grocer (data not shown). We suspect that the combination of shipment, storage, and incubation has a weakening effect on extraembryonic membrane integrity. Regardless, $80 \%$ of embryos that live past the first three days are expected to live at least another week (Auerbach, 1974). After a week, the eggs are expected to live until 16-17 days of gestation; only embryo 7C survived this long.

Since only one embryo lived past day 3 of culturing (day 6 of gestation) we were unable to assess our success rate compared to published data. We would have had to use an unmanageable number of eggs/embryos, for an undergraduate lab, to properly compare our technique to that of Auerbach. Being conservative and assuming no improvement in technique, we would have had to use approximately 30 dozen (360) eggs to obtain an $\mathrm{N}=10$ of embryos surviving past 6 total days of gestation.

Table 3

\begin{tabular}{|c|c|c|c|c|}
\hline \multicolumn{5}{|c|}{ Ex Ovo culturing of embryos: Auerbach Method } \\
\hline Egg & Viability $^{\mathrm{a}}$ (Days) & $\begin{array}{c}\text { Gestation Time }^{\mathrm{b}} \\
\text { (Days) }\end{array}$ & Incubator & Culture Dish \\
\hline $7 \mathrm{~A}$ & 3 & 6 & Hova-Bator & Plastic \\
\hline 7B & 2 & 5 & Hova-Bator & Glass \\
\hline 7C & 13 & 16 & Hova-Bator & Plastic \\
\hline $7 \mathrm{D}$ & Yolk broke & $\mathrm{NA}^{\mathrm{c}}$ & Hova-Bator & Plastic \\
\hline $8 \mathrm{~A}$ & 2 & 5 & Hova-Bator & Glass \\
\hline $8 \mathrm{~B}$ & 1 & 4 & Hova-Bator & Glass \\
\hline $8 \mathrm{C}$ & 1 & 4 & Hova-Bator & Glass \\
\hline $8 \mathrm{D}$ & 1 & 4 & Bacteriological Incubator & Glass \\
\hline $9 \mathrm{~A}$ & 1 & 4 & Bacteriological Incubator & Glass \\
\hline $9 \mathrm{~B}$ & 3 & 6 & Hova-Bator & Glass \\
\hline $9 \mathrm{C}$ & 3 & 6 & Hova-Bator & Glass \\
\hline $9 \mathrm{D}$ & 2 & 5 & Hova-Bator & Glass \\
\hline & & $\begin{array}{r}\text { st-culturing; all en } \\
{ }^{\mathrm{b}} \text { Tota } \\
{ }^{\mathrm{c}} \mathrm{N}\end{array}$ & $\begin{array}{l}\text { were pre-incubated for } 3 \mathrm{~d} \\
\text { tion time } \\
\text { licable }\end{array}$ & \\
\hline
\end{tabular}


A.

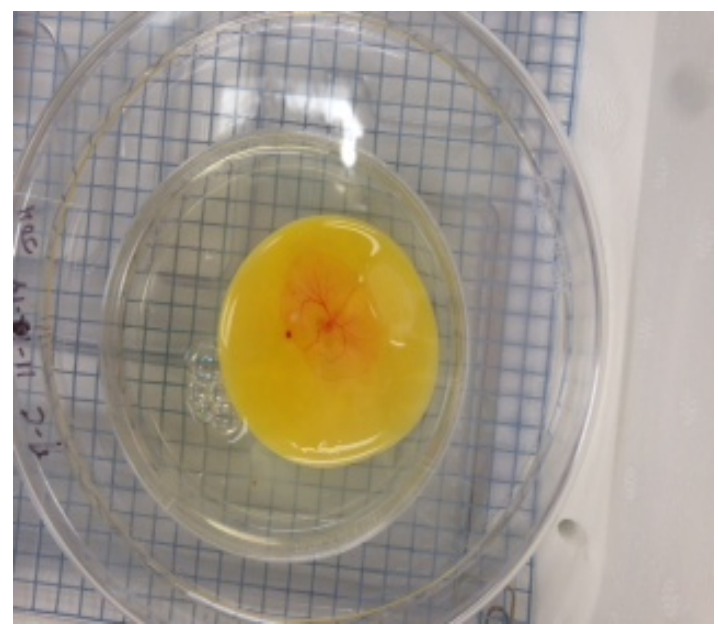

B.

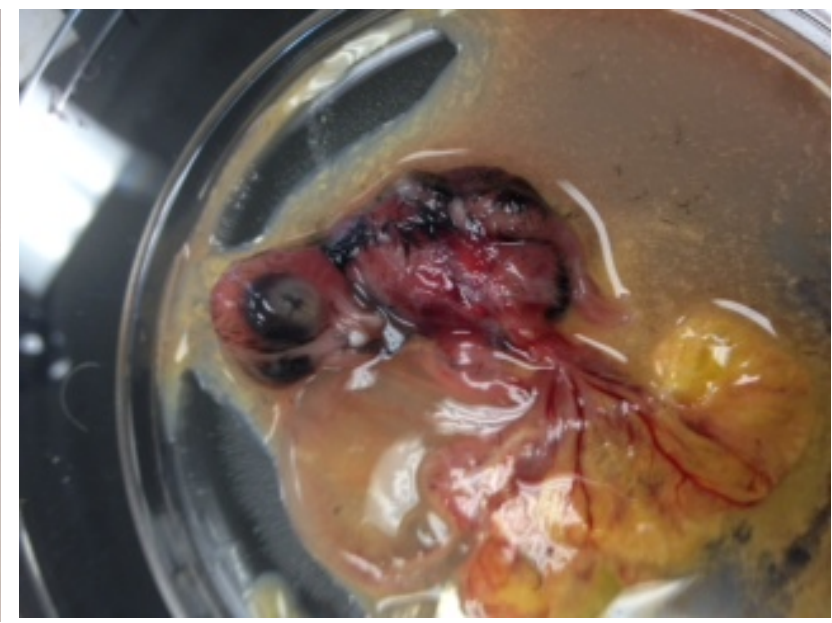

Figure 5. Embryo from Auerbach method of ex ovo culturing. (A) Embryo 7C immediately after being placed in culture dish. Lying in the center of the yolk, the embryo demonstrates optimal orientation. (B) Embryo 7C at 16 days of gestation.

Why ex ovo embryos manipulated on or around day three do not live past a certain gestational stage is not clear. It could be that there are certain environmental factors (e.g. humidity and temperature) required to continue development past this stage. Not all materials in the studies reported (Auerbach, 1974; Schoenwolf, 2009) were available to us. For example, a Hova-Bator was used in place of an actual humidified poultry incubator. Sterile technique was used in the culturing; however, a laminar flow hood, a piece of equipment specifically designed for sterile culturing of cells and embryos, wasn't available. Additionally, in our ex ovo studies, we had to place embryos at room temperature for observations. Temperature and humidity fluctuations during observations could have reduced longevity, though we sought to mitigate this aspect by using short observation times ( $\leq 30 \mathrm{sec}$.). Any of these factors could have contributed to the observed death rate of the embryos. Increased longevity was seen in embryos grown in ovo, as exemplified by the Andacht method (Table 2; Figure 3), most likely due to less exposure to environmental insults and fluctuations. However, for pedagogical purposes the ex ovo culturing technique allows for better observation of embryonic development spatially and temporally (Compare Figure 5 to Figure 1).

\section{Acknowledgements}

R.A.H. thanks J.O.H, my independent research instructor and academic mentor, who provided the means and materials for this study and made several sacrifices for its furtherance. He has taught me that it is never too late to find success through dedication and hard work. J.O.H thanks the Department of Science and Mathematics and the William W. Brady Chair of Science endowment for funding this project.

\section{References}

Andacht, T., Hu, W., \& Ivarie, R. (2004). Rapid and Improved Method for Windowing Eggs Accessing the Stage X Chicken Embryo. Mol. Reprod. Dev. 69(1), 31-4.

Auerbach, R., Kubai, L., Knighton, D., \& Folkman, J. (1974). A Simple Procedure for the Long-Term Cultivation of Chicken Embryos. Dev. Bio. 41(2), 391-94. 
Keller, L., Evans, J., \& Keller, T. (1999). Embryonic Chick Development. Experimental Developmental Biology. San Diego: Academic Press, pp 21-31, 89-95.

Korn, M.J., Cramer K.S. (2007). Windowing Chicken Eggs for Developmental Studies. J. Vis. Exp.(8), 306. http://www.jove.com/index/Details.stp?ID=306, doi:10.3791/306

Gilbert, Scott F. 2010. Developmental Biology, $9^{\text {th }}$ ed. Sinauer Associates, Inc., Sunderland, MA, pp. 431.

Schoenwolf, G. (2009). Hands on Studies. In Vertebrate and Invertebrate Embryos: Guide and Atlas of Descriptive and Experimental Development. Benjamin Cummings, San Francisco, CA, pp. 330-337.

Zareen, N. \& Shahid, U. (2011). Reproducing Windowing Technique for Naked Eye Observation of Chick Embryo Morphogenesis. Ann. Pak. Inst. Med. Sci. 7(3), 146-49. 